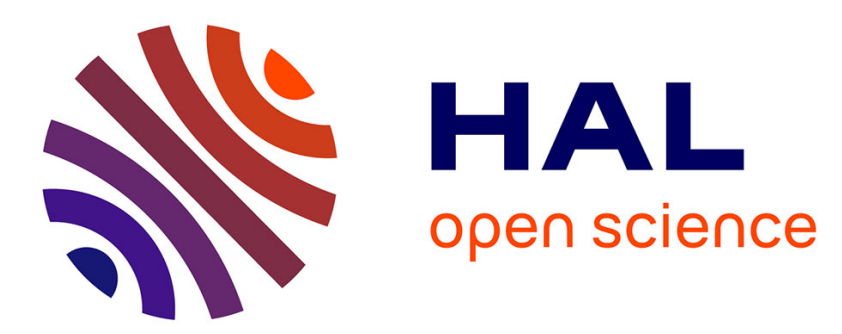

\title{
Differential theory for anisotropic cylindrical objects with an arbitrary cross section \\ Philippe Boyer
}

\section{To cite this version:}

Philippe Boyer. Differential theory for anisotropic cylindrical objects with an arbitrary cross section. Journal of the Optical Society of America. A Optics, Image Science, and Vision, 2013, 30, pp.596 603. hal-00831552

\section{HAL Id: hal-00831552 https://hal.science/hal-00831552}

Submitted on 7 Jun 2013

HAL is a multi-disciplinary open access archive for the deposit and dissemination of scientific research documents, whether they are published or not. The documents may come from teaching and research institutions in France or abroad, or from public or private research centers.
L'archive ouverte pluridisciplinaire HAL, est destinée au dépôt et à la diffusion de documents scientifiques de niveau recherche, publiés ou non, émanant des établissements d'enseignement et de recherche français ou étrangers, des laboratoires publics ou privés. 


\title{
Differential theory for anisotropic cylindrical objects with arbitrary cross-section
}

\author{
Philippe Boyer \\ Département d'Optique P.M. Duffieux, Institut FEMTO-ST, CNRS UMR 6174 Université \\ de Franche-Comté, 25030 Besançon Cedex, France \\ philippe.boyer@univ-fcomte.fr
}

\begin{abstract}
We extend the differential theory to anisotropic cylindrical structures with arbitrary cross-section. Two cases have to be distinguished. When the anisotropic cylinders do not contain the origin, the scattering matrix of the device is calculated from the extended differential theory with the help of the scattering matrix propagation algorithm. The fields outside the cylinders are described by Fourier-Bessel expansions. When the origin is located in one cylinder, the fields inside the cylinder are expressed from a semi-analytical theory related to homogeneous anisotropic medium. In this second case, the formalism of the scattering matrix propagation algorithm is not exactly the same and requires suitable change. The numerical results are in good agreement with the ones obtained for the diffraction by one circular cylinder. The theory is then applied on the diffraction by an elliptical cylinder.

2013 Optical Society of America
\end{abstract}

OCIS codes: $050.1960,160.1190,060.2310$.

\section{Introduction}

For the last half century, the Differential Method (DM) initially developed for studying gratings [1] has been perfomed to modelize a large variety of diffracting devices. It took about thirty years to solve the problems of numerical instabilities due to slow convergences (particularly in TM polarization) and singular matrices to invert occuring when the number of harmonics increases. The first kind of these numerical problems restricts the DM to thin gratings specially in Transverse Magnetic (TM) polarisation [2]. The solution is Fast Fourier Factorization $[3,4]$. It allows faster convergences by using Li's factorization rules [5]. The second kind of numerical instability is related on the divergence of the Maxwell's equation 
integration through very thick gratings. The Scattering Matrix Propagating Algorithm (or S-algorithm) gave the solution in 1996 [6]. From these advances, the DM has been extended to study photonic crystals $[7,8]$ and gratings made of anisotropic and/or non-linear media [9]. Non-periodic objects have recently been studied by the DM written in cylindrical and then spherical coordinate systems. The DM was first applied to classical then conical diffraction by cylindrical objects $[10,11]$, which finally permits modelization of arbitrary cross-section Microstructured Optical Fibers [12]. This numerical method was finally extended to threedimensional structures described in spherical coordinates [13-15].

In this paper, we generalize the DM for cylindrical objets filled with anisotropic media. This method is named the "Anisotropic Differential Method" (ADM). Its formalism requires splitting the space into three regions: two homogeneous regions and the modulated region including the diffracting device (see Fig. 1). The Electromagnetic problem is resolved by applying the boundary conditions at interfaces between these three regions. The DM deals with the integration of Maxwell equations into the modulated area whereas the fields in homogeneous regions are given in analytical expressions. They are expressed by Fourier-Rayleigh expansions in cartesian coordinates or Fourier-Bessel expansions in cylindrical coordinates (assuming isotropic homogeneous media). In cylindrical coordinates, one of the homogeneous areas corresponds to the inner space of a circular cylinder containing the origin as pointed out in Fig. 1. Consequently, modelizing the diffraction of an anisotropic cylinder including the origin induces an inner anisotropic homogenenous area. In view of presenting a general diffracting theory for anisotropic cylinders, we distinguish two cases in our work. When the origin is placed in the anisotropic cylinder, the diffracting problem is resolved by coupling the ADM and the semi-analytical theory stated in [16] called the "Classical Anisotropic Method" (CAM). The ADM integrates Maxwell's equations into the modulated area and the CAM gives the field expressions in the inner anisotropic homogeneous area. For cylinders non containing the origin, only the ADM is used in the modulated area, the fields in homogeneous areas remaining expressed with a Fourier-Bessel expansion (we suppose the ambiant region made of an isotropic homogenenous area).

We first write the CAM equations in a matrix form from which the transmission matrix (T-matrix) of an anisotropic circular cylinder is deduced. This matrix is used in the first step of the S-algorithm when the studied object contains the origin. Then, we show how to compute the T-matrices from the ADM written in cylindrical coordinates. Finally, numerical calculations on circular and elliptical anisotropic cylinders validate the theory by comparison with the works of Monzon et al $[17,18]$. The S-algorithm is always used in order to compute diffracted fields without numerical instabilities. The suitable equations of the S-algorithm for anisotropic media are specially given for the case of anisotropic cylinders containing the origin. 


\section{Presentation of the problem}

We consider an infinitely long cylinder with an axis $O z$ and with an arbitrary directrix $\mathrm{S}$ containing the origin or not. The first case (see Fig. 2) is designated by $\mathbf{C}_{\text {in }}$ and the second case (see Fig. 3) by $\mathbf{C}_{\text {out }}$. The matrix is filled with a homogeneous isotropic medium with absolute permittivity $\epsilon_{m a t}$. The interior region of the cylinder contains an arbitrary (lossy or lossless) anisotropic homogeneous material, characterized by the electric permittivity tensor:

$$
\overline{\bar{\epsilon}}_{c y l}=\left(\begin{array}{ccc}
\overline{\bar{\epsilon}}_{x x} & \overline{\bar{\epsilon}}_{x y} & \overline{\bar{\epsilon}}_{x z} \\
\overline{\bar{\epsilon}}_{y x} & \overline{\bar{\epsilon}}_{y y} & \overline{\bar{\epsilon}}_{y z} \\
\overline{\bar{\epsilon}}_{z x} & \overline{\bar{\epsilon}}_{z y} & \overline{\bar{\epsilon}}_{z z}
\end{array}\right)
$$

in which no symmetry relation is assumed a priori. Only in the $\mathbf{C}_{\text {out-configuration, the }}$ material may be inhomogeneous in the cross-section which implies the dependence of the components of $\overline{\bar{\epsilon}}_{c y l}$ with respect to the cartesian coordinates $x$ and $y$.

By introducing the transformation matrix $\Re$, which links cartesian to cylindrical coordinates, the expression of the permittivity tensor in cylindrical coordinates is given by the relation:

$$
\tilde{\tilde{\epsilon}}_{c y l}=\Re \overline{\bar{\epsilon}}_{c y l} \Re^{T}
$$

where $T$ exponent stands for transpose. The components of $\Re$ are given by the scalar products between the basis vectors $\left\{\mathbf{e}_{x}, \mathbf{e}_{y}, \mathbf{e}_{z}\right\}$ in the cartesian coordinate system and $\left\{\mathbf{e}_{r}, \mathbf{e}_{\theta}, \mathbf{e}_{z}\right\}$ in the cylindrical coordinate system:

$$
\Re=\left(\begin{array}{ccc}
\mathbf{e}_{r} \cdot \mathbf{e}_{x} & \mathbf{e}_{r} \cdot \mathbf{e}_{y} & \mathbf{e}_{r} \cdot \mathbf{e}_{z} \\
\mathbf{e}_{\theta} \cdot \mathbf{e}_{x} & \mathbf{e}_{\theta} \cdot \mathbf{e}_{y} & \mathbf{e}_{\theta} \cdot \mathbf{e}_{z} \\
\mathbf{e}_{z} \cdot \mathbf{e}_{x} & \mathbf{e}_{z} \cdot \mathbf{e}_{y} & \mathbf{e}_{z} \cdot \mathbf{e}_{z}
\end{array}\right)=\left(\begin{array}{ccc}
\cos \theta & \sin \theta & 0 \\
-\sin \theta & \cos \theta & 0 \\
0 & 0 & 1
\end{array}\right)
$$

Since the cylindrical coordinate system is orthogonal, it is not necessary to distinguish between covariant and contravariant tensorial components and thus it will be denoted by subscripts. It is worth noting that while $\tilde{\tilde{\epsilon}}_{i j}$ with $(i, j) \in\{r, \theta, z\}^{2}$ may be dependent on $x$ and $y$, they necessarily depend on $\theta$ through equation (3).

The space is divided into three regions by two circular cylinders with directrices $C_{\min }$ and $C_{\max }$ of radii $R_{\min }$ and $R_{\max }$ respectively defined in order to be tangential to the surface of the studied cylinder. In the case of Fig. 2, the interior region (int) such as $r \leq R_{\min }$ is filled with an anisotopic medium with permittivity tensor $\overline{\bar{\epsilon}}_{\text {int }}=\overline{\bar{\epsilon}}_{c y l}$. The exterior region (ext) such as $r \geq R_{\max }$ is always filled with an isotopic medium with permittivity $\epsilon_{\text {ext }}=\epsilon_{\text {mat }}$. In the case of Fig. 3, the region (int) is filled with the same medium as the region (ext), and $\overline{\bar{\epsilon}}_{\text {int }}=\epsilon_{\text {int }} I_{d}=\epsilon_{\text {ext }} I_{d}$ where $I_{d}$ denotes the identity matrix. The space between the two circular cylinders $C_{\min }$ and $C_{\max }$ is the modulated area. The permittivity tensor of the 
modulated area is noted $\overline{\bar{\epsilon}}(r, \theta)$ of which terms are $2 \pi$-periodic functions with respect to $\theta$ with discontinuities at angles where the cylindrical surface is defined.

An incident planewave in the exterior region with unit amplitude and wavevector $\mathbf{k}^{(i n c)}$ is defined as in ref. [11] by the incident angles $\theta_{\text {inc }}=\left(-\mathbf{e}_{x}, \mathbf{k}_{t}^{(i n c)}\right)$ and $\varphi_{\text {inc }}=\left(\mathbf{k}_{t}^{(i n c)}, \mathbf{k}^{(i n c)}\right)$ where $\mathbf{k}_{t}^{(i n c)}$ is the transverse component of $\mathbf{k}^{(i n c)}$ (in Oxy-plane). The $\mathbf{k}^{(i n c)}$-components in Cartesian coordinates are noted $\left(\alpha_{0}, \beta_{0}, \gamma_{0}\right)$. The polarization vector $\mathbf{E}^{(i n c)}$ of the plane wave can be arbitrarily oriented in the plane transverse to $\mathbf{k}^{(i n c)}$. The $z$-dependence of the scattered field is given by $e^{i \gamma_{0} z}$ with

$$
\gamma_{0}=k_{\text {ext }} \cos \theta_{\text {inc }}
$$

where $k_{\text {ext }}=\left(2 \pi / \lambda_{0}\right) \sqrt{\epsilon_{\text {ext }} / \epsilon_{0}}$ and $\lambda_{0}$ the wavelength in vacuum.

\section{Calculus of the Scattering Matrix of the entire device}

The S-algorithm is an iterative process computing the S-matrix of the modulated area split into $L \in \mathbb{N}$ layers or sub-modulated areas, from T-matrices of each layer. Radii of interfaces surrounding the $(s)$-layers are denoted $r_{s}$, and the T-matrices are now denoted $T^{(s)}$. Precisely, this iterative process propagated along the entire modulated area consists of computing for each layer the $S^{(s+1)}$-matrix on the outermost interface according to the $T^{(s)}$-matrix and the $S^{(s)}$-matrix on the innermost interface [11]. This section provides the calculus of the $T^{(s)}$-matrices of these sub-modulated areas for anisotropic cylinders with an arbitrary crosssection. For the case $\mathbf{C}_{\mathbf{i n}}$, we first present the semi-analytical expression of the T-matrix of an anisotropic circular cylinder required by the initial iteration of the S-algorithm on the first interface $C_{\min }$ and obtained from the theory exposed in ref. [16]. Then, this section continues with the generalization of the Differential Method (DM) to anisotropic cylinders with arbitrary cross-section which permits the calculation of the $T^{(s)}$-matrix of each layer in the modulated area used in the S-algorithm.

\section{A. Transmission matrix of an anisotropic circular cylinder}

We consider here a circular anisotropic cylinder with radius $R$ centered to the origin, as described in Fig. 2 in the particular case of a non modulated area, i.e. $R_{\min }=R_{\max }=R$. We first write in a matrix form the expressions of the electromagnetic field in the Fourier space for the anisotropic medium from the basic equations of the CAM presented in ref. [16]. The transmission matrix of the cylinder is then obtained by applying the electromagnetic continuities to its interface $r=R$ with the analytical field expressions for the isotropic medium outside the cylinder.

In the anisotropic medium $(r \leq R)$, the equations (38), (39), (42) and (43) in ref. [16] allow to express the Fourier coefficients of $\theta$ and $z$-components of the electromagnetic field 
versus their undetermined amplitudes, in a matrix form:

$$
[F(r)]=\widetilde{\Psi}(r)[\widetilde{A}]
$$

where the column matrix $[F(r)]$ is made of $\left[E_{\theta}\right],\left[E_{z}\right],\left[H_{\theta}\right]$ and $\left[H_{z}\right]$, then the column matrix $[\widetilde{A}]$ contains the amplitudes $\widetilde{A}_{1, \nu}$ and $\widetilde{A}_{2, \nu}$ in the anisotropic media. We use the same notation as shown in $[11]$ that introduces $[U]$ as a column matrix containing the truncated series of the quantity $U$. The matrix $\widetilde{\Psi}$ is made of $4 \times 2$ blocks:

$$
\widetilde{\Psi}(r)=\left(\begin{array}{cc}
\widetilde{\Psi}_{11}(r) & \widetilde{\Psi}_{12}(r) \\
\widetilde{\Psi}_{21}(r) & \widetilde{\Psi}_{22}(r) \\
\widetilde{\Psi}_{31}(r) & \widetilde{\Psi}_{32}(r) \\
\widetilde{\Psi}_{41}(r) & \widetilde{\Psi}_{42}(r)
\end{array}\right)
$$

in which

$$
\begin{gathered}
\left\{\widetilde{\Psi}_{1 j}(r)\right\}_{n, \nu}=\frac{n}{r k_{j, \nu, \rho}}\left(\alpha_{n, j, \nu}-\frac{\beta_{n, j, \nu}}{k_{j, \nu, \rho}}\right) J_{n}\left(k_{j, \nu, \rho} r\right)-\frac{\beta_{n, j, \nu}}{k_{j, \nu, \rho}} J_{n+1}\left(k_{j, \nu, \rho} r\right) \\
\left\{\widetilde{\Psi}_{2 j}(r)\right\}_{n, \nu}=\gamma_{n, j, \nu} J_{n}\left(k_{j, \nu, \rho} r\right) \\
\left\{\widetilde{\Psi}_{3 j}(r)\right\}_{n, \nu}=\frac{-1}{i \omega \mu_{0}}\left[\frac{n}{r k_{j, \nu, \rho}}\left(k_{j, \nu, \rho} \gamma_{n, j, \nu}+\frac{\beta_{n, j, \nu} \gamma_{0}}{k_{j, \nu, \rho}}-\alpha_{n, j, \nu} \gamma_{0}\right) J_{n}\left(k_{j, \nu, \rho} r\right)+\left(\alpha_{n, j, \nu} \gamma_{0}-k_{j, \nu, \rho} \gamma_{n, j, \nu}\right) J_{n+1}\left(k_{j, \nu, \rho} r\right)\right] \\
\left\{\widetilde{\Psi}_{4 j}(r)\right\}_{n, \nu}=\frac{\beta_{n, j, \nu}}{i \omega \mu_{0}} J_{n}\left(k_{j, \nu, \rho} r\right)
\end{gathered}
$$

with $j=1$ or 2 and using the formula $J_{n}^{\prime}(\zeta)=n J_{n}(\zeta) / r-J_{n+1}(\zeta) \cdot \omega$ is the incident field circular frequency and $\mu_{0}$ the vacuum permeability. We precise that coefficients $\alpha_{n, j, \nu}$, $\beta_{n, j, \nu}$ and $\gamma_{n, j, \nu}$ are respectively given by equations (33) to (35) in ref. [16], and $k_{j, \nu, \rho}$ is the $\rho$-component of the wavevectors $\mathbf{k}_{j}(\varphi)$ with a discretization of angle $\varphi$ on $2 \pi$-range (see equations (15) and (16) in ref. [16]). We notice that the size of the matrix $\widetilde{\Psi}(r)$ is $4(2 N+1) \times 2 N_{\varphi}$ where $N$ denotes the truncated order of the Fourier developments so that $n \in[-N,+N]$ and $N_{\varphi}$ the step number of $\varphi$-discretization so that $\nu \in\left[1, N_{\varphi}\right]$.

In the outer isotropic region $(r \geq R)$, the column matrix $[F(r)]$ is linked to the column ma$\operatorname{trix}\left[V^{(e x t)}(r)\right]$ containing the quantities $A_{e, n}^{(e x t)} J_{n}\left(k_{t, e x t} r\right), A_{h, n}^{(e x t)} J_{n}\left(k_{t, e x t} r\right), B_{e, n}^{(e x t)} H_{n}^{+}\left(k_{t, e x t} r\right)$ and $B_{h, n}^{(e x t)} H_{n}^{+}\left(k_{t, e x t} r\right)$ by the relation (34) in ref. [11] that we write again:

$$
[F(r)]=\Psi^{(e x t)}(r)\left[V^{(e x t)}(r)\right]
$$

with $k_{t, e x t}^{2}=k_{e x t}^{2}-\gamma_{0}^{2}$ and $k_{e x t}^{2}=\omega^{2} \mu_{0} \epsilon_{e x t}$. The terms $A_{e, n}^{(e x t)}, A_{h, n}^{(e x t)}, B_{e, n}^{(e x t)}$ and $B_{h, n}^{(e x t)}$ are the Fourier amplitudes of the field $z$-components in the (ext) region. The matrix $\Psi^{(e x t)}(r)$ is explicitly given by equations (34) to (37) in the same previous reference. 
Finally, the transmission matrix of the anisotropic circular cylinder denoted $T^{(a n i)}$ is obtained by writing the continuity of $[F(r)]$ at the interface $r=R$ :

$$
\left[V^{(e x t)}(R)\right]=T^{(a n i)}[\widetilde{A}]
$$

where

$$
T^{(a n i)}=\left\{\Psi^{(e x t)}(R)\right\}^{-1} \widetilde{\Psi}(R)
$$

in which the inverse of the diagonal-block matrix $\Psi^{(e x t)}(R)$ is analytically expressed. The size of this matrix is $4(2 N+1) \times 2 N_{\varphi}$. We remark that the unknown quantities are the diffracted amplitudes $B_{e, n}^{(e x t)}, B_{h, n}^{(e x t)}, \widetilde{A}_{1, \nu}$ and $\widetilde{A}_{2, \nu}$ whereas $A_{e, n}^{(e x t)}, A_{h, n}^{(e x t)}$ are the given incident ones. The resolution of the diffracting problem by using the equation (12) requires that the discretization with respect to $\varphi$ follows the same truncation of the Fourier series, i.e. $N_{\varphi}=2 N+1$.

\section{B. Differential theory for anisotropic cylinders}

We pose here the main equations of the ADM which lead to the T-matrix expression of one anisotropic modulated area. They may be seen as the achievement of the Differential theory stated in ref. [11] to anisotropic cylinders with arbitrary cross-section. The last formulation of this theory taking into account the Li factorization rules in the linear relation between $\mathbf{D}$ and $\mathbf{E}$ (called the Fast Fourier Factorization method or FFF) was initially written for the general case of periodic objects in Cartesian coordinates and made of inhomogeneous and anisotropic materials $[2,4]$.

In the modulated area, the permittivity tensor may be written as a function of the radial and angular variables: $\tilde{\tilde{\epsilon}}(r, \theta)$, considering the coordinate transformation matrix $\Re$ (see equation (2)) and the alternation of tensors $\overline{\bar{\epsilon}}_{\text {mat }}$ and $\overline{\bar{\epsilon}}_{c y l}$ versus $\theta$ for a fixed $r$-value. In the Fourier space, the FFF method consists of expressing the column matrix $[\mathbf{D}]$ as the product between $Q_{\epsilon}$ matrix (equations (14-15) in ref. [11]) and the column matrix [E], $Q_{\epsilon}$ depending on $C_{\epsilon}$ matrix (equation (12) in ref. [11]). The independence of the object according to $z$ implies that $C_{\epsilon}$ is written for inhomogeneous and anisotropic media as:

$$
C_{\epsilon}=\frac{1}{\delta}\left(\begin{array}{ccc}
N_{r} \epsilon_{r \theta}+N_{\theta} \epsilon_{\theta \theta} & N_{r} & -N_{r}\left(N_{r} \epsilon_{r z}+N_{\theta} \epsilon_{\theta z}\right) \\
-N_{r} \epsilon_{r r}+N_{\theta} \epsilon_{\theta r} & N_{\theta} & -N_{\theta}\left(N_{r} \epsilon_{r z}+N_{\theta} \epsilon_{\theta z}\right) \\
0 & 0 & \delta
\end{array}\right)
$$

where

$$
\delta=N_{r}^{2} \epsilon_{r r}+N_{\theta}^{2} \epsilon_{\theta \theta}+N_{r} N_{\theta}\left(\epsilon_{\theta r}+\epsilon_{r \theta}\right)
$$

in which $\epsilon_{i j}$ are the components of tensor $\tilde{\tilde{\epsilon}}(r, \theta)$. All terms $N_{i}$ and $\epsilon_{i j}$ with $(i, j) \in\{r, \theta, z\}^{2}$ are dependent on $r$ and $\theta$. We specify that the functions $N_{i}$ are defined as arbitrary extensions of the components of the normal vector to the diffracting surface $(S)$ inside the whole modulated 
area (see ref. [10] in which different kinds of extensions are analyzed). $N_{i}$ functions are chosen here as piecewise-constant functions with respect to $\theta$. However, the non trivial dependence of $C_{\epsilon}$ terms to $r$ and $\theta$ imposes the use of a Fast Fourier Transformation algorithm to compute its Fourier coefficients from which the matrix $Q_{\epsilon}$ is deduced. The equation (14) implies that the $Q_{\epsilon}$ matrix is made a priori of 9 non nil blocks since only two terms of $C_{\epsilon}$ are nil, when it contains only 5 non nil blocks for isotropic media [11]. Combining the Maxwell equations and constitutive relations of media expressed thanks to equations (14) leads to the differential equation sets given by eqs. (26)-(27) in ref. [11]. Finally, the $T^{(s)}$-matrix of the anisotropic modulated area is obtained by the integration of this differential set using a shooting method along each $(s)$-layer (see section 4.C in [11]):

$$
T^{(s)}=\left\{\Psi^{(e x t)}\left(r_{s+1}\right)\right\}^{-1}\left[F_{\text {integ }}\left(r_{s+1}\right)\right] \Psi^{(e x t)}\left(r_{s}\right)
$$

where $\left[F_{\text {integ }}\left(r_{s+1}\right)\right]$ is the vector $[F(r)]$ obtained at the end of the integration (initial vector chosen equal to identity matrix).

Before to numerically validate the theory, it is worth to mention the calculation of the $T^{(s)}$-matrices of an isotropic and homogeneous $(s)$-layer. Its permittivity is denoted $\epsilon_{i s o}$. The $T^{(s)}$-matrix is easily expressed by applying the continuity of $[F(r)]$ at interfaces $r=r_{s}$ and $r_{s+1}$ and with the equation (11) written for the isotropic medium (iso):

$$
T^{(s)}=\left\{\Psi^{(e x t)}\left(r_{s+1}\right)\right\}^{-1} \Psi^{(i s o)}\left(r_{s+1}\right) C^{(i s o)}\left(r_{s}, r_{s+1}\right)\left\{\Psi^{(i s o)}\left(r_{s}\right)\right\}^{-1} \Psi^{(e x t)}\left(r_{s}\right)
$$

with

$$
C^{(i s o)}\left(r_{s}, r_{s+1}\right)=\left(\begin{array}{cccc}
J & 0 & 0 & 0 \\
0 & J & 0 & 0 \\
0 & 0 & H & 0 \\
0 & 0 & 0 & H
\end{array}\right)
$$

and

$$
\begin{aligned}
(J)_{n, m} & =\frac{J_{n}\left(k_{t, i s o} r_{s+1}\right)}{J_{n}\left(k_{t, i s o} r_{s}\right)} \delta_{n m} \\
(H)_{n, m} & =\frac{H_{n}^{+}\left(k_{t, i s o} r_{s+1}\right)}{H_{n}^{+}\left(k_{t, i s o} r_{s}\right)} \delta_{n m}
\end{aligned}
$$

$\delta_{n m}$ is the Kronecker symbol.

\section{Numerical validation}

We present in this section the validation results of ADM and the coupling between ADM and CAM. To accomplish this, the numerical results are compared with the ones shown by Monzon et al in $[17,18]$ and by our previous works in [16] treating the diffraction of a planewave by an anisotropic circular cylinder. At first, the study of one anisotropic circular 
cylinder non containing the origin permits the validation of only the ADM. After briefly introducing the suitable equations of S-algorithm for cylinders containing the origin, we have extended this geometry to elliptical anisotropic cylinders which has required coupling ADM and CAM. Convergence tests reinforce the numerical analysis. The numerical code is written in Fortran 90 and is executed on a personal computer with CPU at 2.66 GHz. The length units are not mentioned since no material dispersion is considered (fixed refractive index).

\section{A. $\mathbf{C}_{\text {out }}$-case}

As shown in Fig. 3, we are interested in an anisotropic cylinder that does not contain the origin $\left(\mathbf{C}_{\text {out-case }}\right)$, particularly in a circular cylinder defined by a center $C$, a radius $R=1$ and a distance to origin $\Lambda=2$ (see Fig. 4). In spite of this common profile, this geometrical mounting is complex to compute with ADM (the need of many Fourier coefficients to describe the profile) on account of non angular symmetry, but remains relevant to validate the theory. The parameters of the incident planewave are $\lambda=2, \varphi_{i n c}=90^{\circ}$ and $\theta_{i n c}=30^{\circ}$. Later on, we suppose that any permittivity tensor is diagonal, i.e. the optic axes are parallel to the Cartesian axis. The first studied cylinder is made of a biaxial anisotropic medium with relative permittivities equal to $\epsilon_{x x}=2, \epsilon_{y y}=2.25$ and $\epsilon_{z z}=2.5$. The outside region is filled with air $\left(\epsilon_{\text {mat }}=1\right)$. We compare the numerical results obtained with the ADM for the cylinder non including the origin (fig. 4) and the ones obtained with the CAM for the same cylinder but centered to origin. The CAM is considered as the reference method due to its large number of numerical accuracies (theory based on semi-analytical formulation) and to the fact that it has been widely validated in [16]. It is important to notice that the well-known S-algorithm [6,11] is used in order to avoid numerical instabilities. Fig. 5(a) points out the electric and magnetic differential cross sections (DCS), expressed in eqs. (67) and (68) in [16], noted $\sigma_{E}(\theta)$ and $\sigma_{H}(\theta)$ respectively, and calculated with both methods. For CAM, the truncation order $N$ is equal to 20. For ADM, $N=100$ and the number $L$ of layers for S-algorithm is equal to 40. Good agreement between the results of CAM and ADM is confirmed by convergence tests shown in Fig. 5(b) of $\sigma_{H}\left(270^{\circ}\right)$ and $\sigma_{E}\left(301^{\circ}\right)$ according to $N$. The relative error between $\sigma_{H}\left(270^{\circ}\right)$ computed at $N=100$ for ADM and at $N=20$ for CAM reaches $0.03 \%$, and is equal to $0.4 \%$ for $\sigma_{E}\left(301^{\circ}\right)$. It is worth noticing that the incident polarization and the cylinder electrical size can affect the convergence speed. For instance, the convergence slows down when the circular cylinder radius decreases. We have also checked that the diffracted field maps of $\left|E_{z}\right|$ and $\left|H_{z}\right|$ obtained with ADM for $N=60$, and illustrated in Fig. 6, are exactly the same as the ones computed with CAM.

We consider now the circular cylinder with the same geometrical parameters but filled with a uniaxial medium such as $\epsilon_{x x}=4.87526$ and $\epsilon_{y y}=\epsilon_{z z}=5.29$ for which the DCS are 
illustrated in Fig. 7(a). The gaps between the permittivities inside and outside the cylinder are clearly higher than the ones in the previous case of a biaxial medium, that is why the ADM results converge more slowly to the value obtained with CAM (see Fig. 7(b)): the relative error between $\sigma_{H}\left(270^{\circ}\right)$ computed at $N=100$ for $\mathrm{ADM}$ and at $N=20$ for CAM reaches $1.39 \%$, and is equal to $0.66 \%$ for $\sigma_{E}\left(321^{\circ}\right)$. We would like to specify that the computation time is 11 seconds at $N=10$ and 174 minutes at $N=100$ for calculation with ADM whatever the anisotropic medium.

\section{B. $\mathbf{C}_{\mathbf{i n}}$-case}

Above all, we have to focus our attention on the S-algorithm for $\mathbf{C}_{\mathbf{i n}}$-case. The region (int) is filled with the anisotropic medium for which the Fourier coefficients of the field are expressed from the CAM (Eq. (5)). The modulated area is split into $L \in \mathbb{N}$ layers $(s), s \in[1, L]$, with $T^{(s)}$-matrices given by equation (16). An infinitely thin isotropic layer with permittivity $\epsilon_{\text {ext }}$ is added at each interface (with radius $r_{s}$ ). For a fixed thickness $R_{\max }-R_{\min }$, the number $L$ of layers is chosen so that the matrices $T^{(s)}$ are well conditioned. In other words, the thicknesses $r_{s+1}-r_{s}$ of $(s)$-layers are chosen small enough to avoid numerical instabilities (see also ref. [2]). To maintain precision on surface profile discretization, the number $M$ of steps in differential set's integration is chosen such as $M \times L$ remains constant. For the coupling ADM-CAM, the $S^{\left(s^{\prime}\right)}$-matrix at the interface $r=r_{s^{\prime}}\left(s^{\prime} \in[1, L+1]\right)$ takes the following form:

$$
\left(\begin{array}{c}
{\left[B^{(e x t)}\left(r_{s^{\prime}}\right)\right]} \\
{[\widetilde{A}]}
\end{array}\right)=S^{\left(s^{\prime}\right)}\left[A^{(e x t)}\left(r_{s^{\prime}}\right)\right]
$$

where the column matrix $\left[B^{(e x t)}(r)\right]$ contains the components $A_{e, n}^{(e x t)} J_{n}\left(k_{t, e x t} r\right)$ and $A_{h, n}^{(e x t)} J_{n}\left(k_{t, e x t} r\right)$, then $\left[A^{(e x t)}(r)\right]$ the components $B_{e, n}^{(e x t)} H_{n}^{+}\left(k_{t, e x t} r\right)$ and $B_{h, n}^{(e x t)} H_{n}^{+}\left(k_{t, e x t} r\right)$. The matrix $S^{\left(s^{\prime}\right)}$ differs from the common one used in S-algorithm $[6,11]$ since it is a non square matrix with a size of $4(2 N+1) \times 2(2 N+1)$. Besides, it links two different kinds of amplitudes: $[\widetilde{A}]$ and $\left[A^{(e x t)}\left(r_{s^{\prime}}\right)\right]$. Noticing $S_{1}^{\left(s^{\prime}\right)}$ and $S_{2}^{\left(s^{\prime}\right)}$ both $2(2 N+1) \times 2(2 N+1)$ blocks of $S^{\left(s^{\prime}\right)}$, the iterative process of the S-algorithm reduces here to:

$$
\begin{gathered}
Z^{(s)}=\left[T_{11}^{(s)}+T_{12}^{(s)} S_{1}^{(s)}\right]^{-1} \\
S_{2}^{(s+1)}=S_{2}^{(s)} Z^{(s)} \\
S_{1}^{(s+1)}=\left[T_{21}^{(s)}+T_{22}^{(s)} S_{1}^{(s)}\right] Z^{(s)}
\end{gathered}
$$

The initial matrix $S^{(1)}$ at the interface $r=r_{1}=R_{\text {min }}$ is obtained thanks to the $T^{(a n i)}$-matrix given by equation (13). By comparison of the equation (12) with $R=R_{\min }$ and equation (21) for $s^{\prime}=1$, we obtain $S_{2}^{(1)}=\left\{T_{1}^{(a n i)}\right\}^{-1}$ and $S_{1}^{(1)}=T_{2}^{(a n i)}\left\{T_{1}^{(a n i)}\right\}^{-1}$ using the same 
notation for blocks of $T^{(a n i)}$ as the $S^{\left(s^{\prime}\right)}$ 's ones. At the last interface $r=r_{L+1}$, the $S^{(L+1)}$ matrix identifies to the $S$-matrix of the entire modulated area defined in equation (21) with $\left[B^{(e x t)}\left(r_{L+1}\right)\right]=\left[B^{(e x t)}\left(R_{\max }\right)\right]$ and $\left[A^{(e x t)}\left(r_{L+1}\right)\right]=\left[A^{(e x t)}\left(R_{\max }\right)\right]$.

We study in this section the diffraction by an elliptical cylinder centered to origin $\left(\mathbf{C}_{\mathbf{i n}}{ }^{-}\right.$ case) and filled with the same biaxial medium as the previous one. The modelization of such a device requires coupling the CAM (to describe the fields in region (int)) with the ADM (to describe the fields in the modulated area). We observe in Fig. 8(a) the variation of DCS when the semi-major axis of length $a$ (along the x-axis) increases from 1 to 1.5, the semi-minor axis of length $b$ (along the y-axis) being fixed at 1 . The continuity of variation of $\sigma_{H}\left(270^{\circ}\right)$ and $\sigma_{H}\left(90^{\circ}\right)$ versus $a$ for a circular cylinder is checked in viewing Fig. 8(b). We show that the increase of the semi-major axis implies a reinforcement of the transmitted peak and an attenuation of the reflected peak.

But we tarry on the convergence test of the coupling ADM-CAM versus the truncation order as shown in Fig. 9 for the highest point $\sigma_{H}\left(270^{\circ}\right)$. In fact, thanks to a semi-analytical formulation given in eqs. (6) to (10), the CAM converges quickly according to the truncation order $N$ but remains numerically unstable. The terms of matrix $\widetilde{\Psi}(r)$ are linear depending on Bessel functions $J_{n}\left(k_{j, \nu, \rho}\right)$ which tend toward zero when $n$ increases. Consequently, few columns of $\widetilde{\Psi}(r)$ and $T^{(a n i)}$ contain terms with very low values if $N$ is sufficiently high. The inversion of $T_{1}^{(a n i)}$ needed to calculate the S-matrix at the first step of the S-algorithm, becomes numerically tricky (determinant of $T^{(\text {ani) }}$ tends toward zero). We specify that these numerical instabilities do not occur in the case of the T-matrix for an isotropic layer (eq. (17)) because the terms of $\Psi^{(j)}(r)$ are normalized by the Bessel and Hankel functions (see eqs (19) and (20) then eqs (35) to (37) in ref. [11]). So, only the blocks $T_{11}^{(s)}$ may diverge when $N$ and the layer's thickness increases, which justifies the use of the S-algorithm (the terms of other blocks have values close to unity). For anisotropic media, the matrix $\widetilde{\Psi}(r)$ cannot be normalized in the same way because the subscripts $n$ and $\nu$ are basically different. One idea consists of normalizing all terms by $J_{N}\left(k_{j, \nu, \rho}\right)$ ( $n$ fixed) but this implies a divergence of both blocks of $T^{(a n i)}$ (not only $T_{1}^{(a n i)}$ ) for terms evaluated at low values of $n$. Finally, we have found no solution to avoid numerical instabilities that occur in the inversion of $T_{1}^{(a n i)}$ for high values of $N$.

However, the CAM is very accurate before numerical instabilities appear, and it converges faster than ADM. That is why it seems judicious for coupling both methods to impose different truncation orders noted $N_{C A M}$ and $N_{A D M}$ such as $N_{C A M} \leq N_{A D M}$. When $N_{A D M}$ is strictly higher than $N_{C A M}$, the matrix $S^{(1)}$ is computed at $N=N_{C A M}$ (from the matrix $T^{(a n i)}$ ) and permits the evaluation of $S^{(1)}$ at $N=N_{A D M}$, the other terms for high orders being forced to zero. This last matrix becomes the initial matrix of the S-algorithm (equal to the identity matrix otherwise). This operation may be seen as a filtering procedure to order 
$N_{C A M}$ of all Fourier expansions in CAM when the truncation order in ADM is fixed to $N_{A D M}$. For instance, Fig. 9 clearly points out that the coupling between ADM and CAM is wrongly

conditioned from $N=N_{C A M}=N_{A D M}=20$ only due to the divergence of $\left\{T^{(a n i)}\right\}^{-1}$ in CAM. But numerical instabilities progressively disappear when $N_{C A M}$ decreases (see subfigure in Fig. 9).

\section{5. conclusion}

We have validated the Differential Method for studying diffraction by the anisotropic cylinder. The homogeneous regions, induced by the space splitting, impose consideration of two kinds of configurations with regard to origin localization. When the origin is placed outside the anisotropic objet, the method uses a generalized form of the differential method equations. But, in the opposite case, this extended differential method is combined with a semi-analytical one describing the field in one anisotropic homogeneous region. The suitable relations to propagate fields in modulated area by the S-algorithm are thus exposed in this case. The numerical application of the method on an anisotropic circular cylinder is in good agreement with results obtained by Monzon et al $[17,18]$. The case of an anisotropic elliptical cylinder is then studied. It reveals that a balanced convergence requires two different harmonic numbers of both truncated developments of the combined methods.

Our future works will deal with finding modes in optical fibers made of anisotropic media by using the present methods. In fact, one of the advantages of the methods is to consider an anisotropic cylinder with an arbitrary cross-section. So, we will be particularly interested in liquid crystal photonic crystal fibers. They are particularly interesting since their properties of index-guiding may be tuned by this kind of anisotropic material. The method will be reinforced by the consideration of symmetry.

Acknowledgements: I would like to thank Donna L'Hôte from the Centre de linguistique appliquée (CLA) of Besançon (France) for her helpful advice.

\section{References}

1. G. Cerutti-Maori and R. Petit and M. Cadilhac. Etude numrique du champ diffract par un rseau. C. R. Ac. Sc. Paris., 268:1060-1063, 1969.

2. M. Nevière and E. Popov. Light propagation in periodic media ; differential theory and design. Marcel Dekker, New York, Basel and Honk Kong., 2003.

3. E. Popov and M. Nevière. Grating theory: New equations in Fourier space leading to fast converging result in TM polarization. J. Opt. Soc. Am. A., 17:1773-1784, 2000. 
4. E. Popov and M. Nevière. Maxwell equations in Fourier space: fast-converging formulation for diffraction by arbitrary shaped, periodic, anisotropic media. J. Opt. Soc. Am. A., 18:2886-2894, 2001.

5. L. Li. Use of Fourier series in the analysis of discontinuous periodic structures. J. Opt. Soc. Am. A., 13:1870-1876, 1996.

6. L. Li. Formulation and comparison of two recursive matrix algorithms for modeling layered diffraction gratings. J. Opt. Soc. Am. A., 13:1024-1035, 1996.

7. S. Enoch and E. Popov and M. Nevière 3-D photonic crystals dispersion relation: improved convergence using fast Fourier factorization (FFF) method. Proc. SPIE., 4438:183-190, 2001.

8. S. Enoch and E. Popov and N. Bonod Analysis of the physical origin of surface modes on finite size photonic crystals. Phys. Rev. B., 72, 2005.

9. N. Bonod and E. Popov and M. Nevière Factorization of nonlinear Maxwell equations in periodic media. Application to the optical Kerr effect. Opt. Commun., 244:389-398, 2005.

10. P. Boyer and E. Popov and M. Nevière and G. Tayeb Diffraction theory in TM polarization: application of the fast Fourier factorization method to cylindrical devices with arbitrary cross section. J. Opt. Soc. Am. A, 21:2146-2153, 2004.

11. P. Boyer and E. Popov and M. Nevière and G. Renversez Diffraction theory: Application of the fast Fourier factorization to cylindrical devices with arbitrary cross lighted in conical mounting. J. Opt. Soc. Am. A., 23:1146-1158, 2006.

12. P. Boyer AND G. Renversez AND E. Popov AND M. Nevière Improved differential method for microstructured optical fibres. J. Opt. A: Pure Appl. Opt., 9:1-13, 2007.

13. B. Stout and M. Nevière and E. Popov Light diffraction by a three-dimensional object: differential theory. J. Opt. Soc. Am. A, 22:2385-2404, 2005.

14. B. Stout and M. Nevière and E. Popov Mie scattering by an anisotropic object. Part I: homogeneous sphere. J. Opt. Soc. Am. A, 23:1111-1123, 2006.

15. B. Stout and M. Nevière and E. Popov Mie scattering by an anisotropic object. Part II: arbitrary-shaped object - differential theory. J. Opt. Soc. Am. A, 23:1124-1134, 2006.

16. M. Nevière and E. Popov and P. Boyer Diffraction theory of an anisotropic circular cylinder. J. Opt. Soc. Am. A, 23:1731-1740, 2006.

17. J.C. Monzon and N.J. Damaskos Two-dimensional scattering by homogeneous anisotropic rod. IEEE Trans. Antennas Propagat., 34:1243-1249, 1986.

18. J.C. Monzon Three-dimensional scattering by an infinite homogeneous anisotropic circular cylinder: A spectral approach. IEEE Trans. Antennas Propagat., 35:670-682, 1987. 


\section{List of Figure Captions}

Fig. 1. (Color online) Schematic representation of two kinds of anisotropic cylinders studied. Fig. 2. (Color online) Configuration $\mathbf{C}_{\mathbf{i n}}$ : Anisotropic cylinder containing the origin.

Fig. 3. (Color online) Configuration $\mathbf{C}_{\mathbf{o u t}}$ : Anisotropic cylinder non containing the origin.

Fig. 4. (Color online) Anisotropic circular cylinder non containing the origin.

Fig. 5. (Color online) Comparison between DCS calculated with CAM and ADM for a biaxial medium.

Fig. 6. (Color online) Diffracted field maps for anisotropic circular cylinder computed with ADM for $N=60 . \tau$ is the relative error between CAM and MDA field maps (average relative error computed at each point of field maps).

Fig. 7. (Color online) Comparison between DCS calculated with CAM and ADM for an unaxial medium.

Fig. 8. (Color online) Study of DCS for anisotropic elliptical cylinders with different values of the semi-major axis $a$. The semi-minor axis $b$ is fixed at 1 .

Fig. 9. (Color online) $\sigma_{H}\left(270^{\circ}\right)$ versus $N_{A D M}$ for different values of $N_{C A M}$ and for $a=1.1$. 


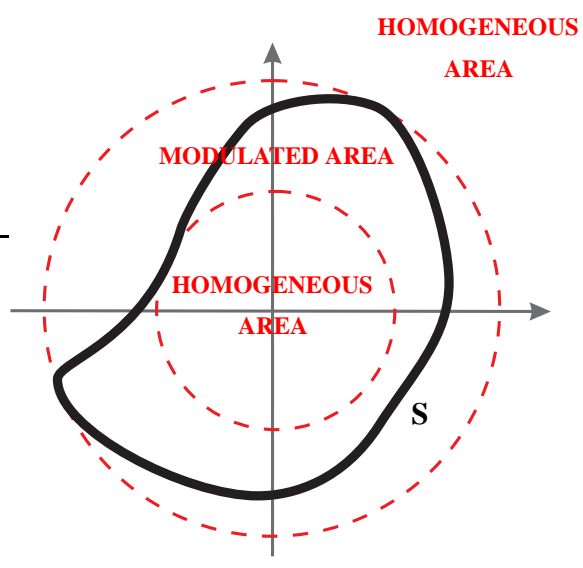

Cylinder containing the origin

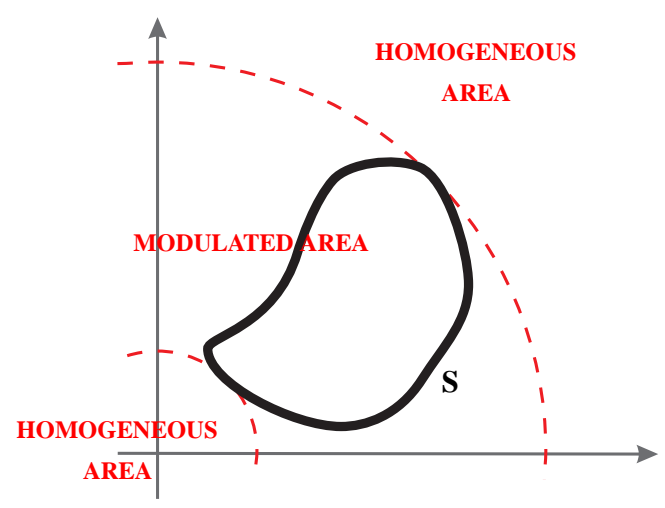

Cylinder non containing the origin

Fig. 1. (Color online) Schematic representation of two kinds of anisotropic cylinders studied. 


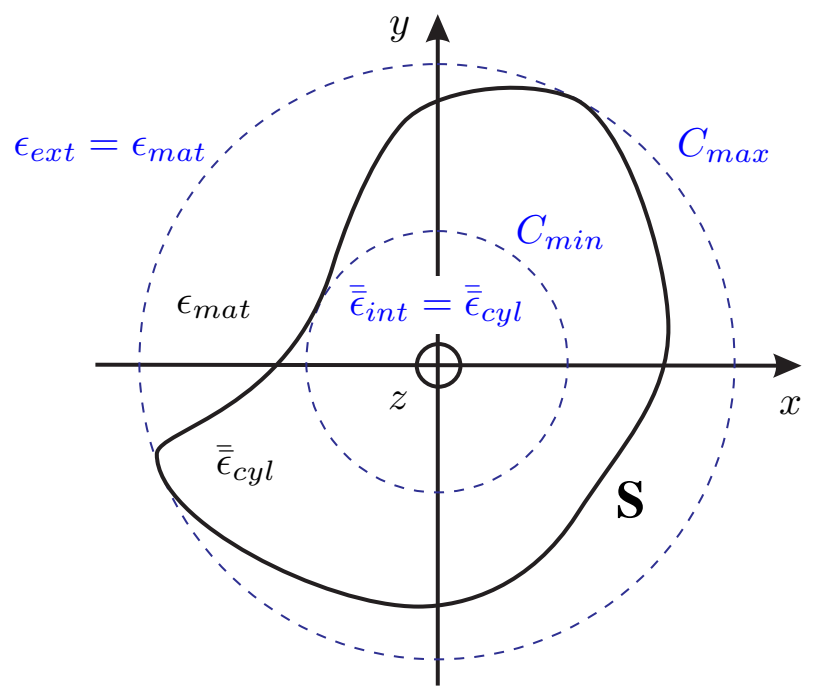

Fig. 2. (Color online) Configuration $\mathbf{C}_{\mathbf{i n}}$ : Anisotropic cylinder containing the origin. 


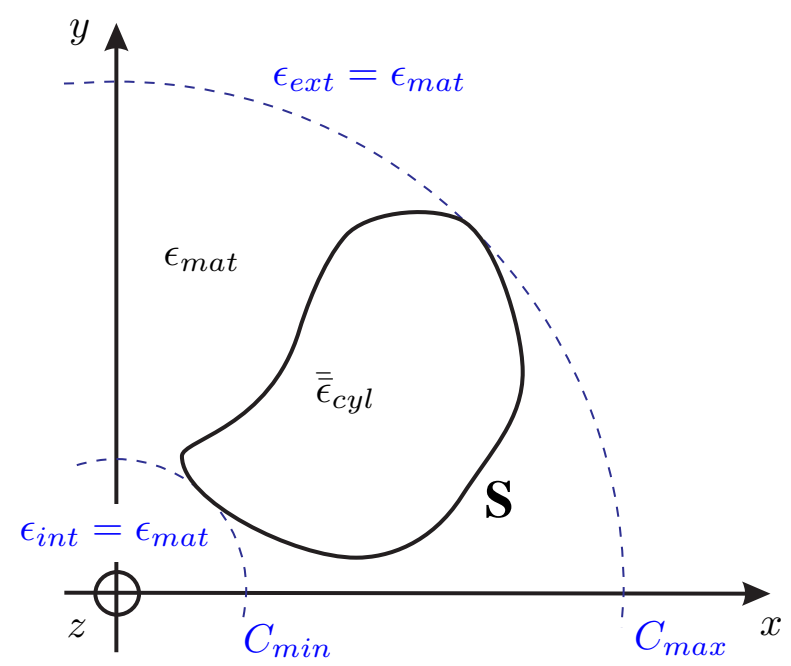

Fig. 3. (Color online) Configuration $\mathbf{C}_{\text {out }}$ : Anisotropic cylinder non containing the origin. 


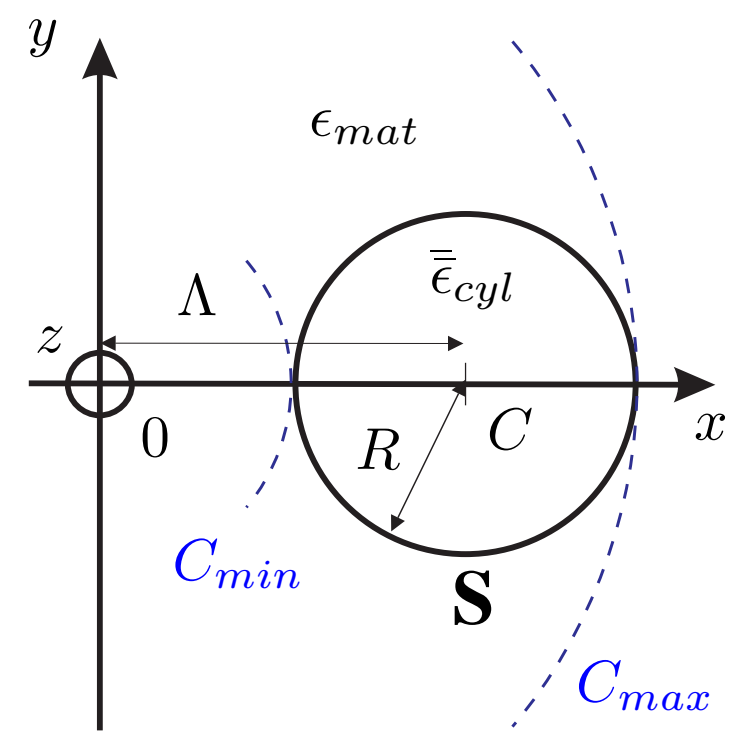

Fig. 4. (Color online) Anisotropic circular cylinder non containing the origin. 


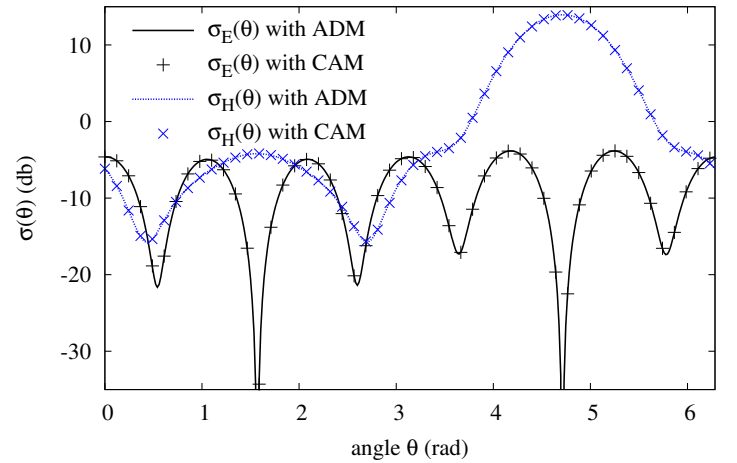

(a) DCS versus angle for electric and magnetic fields computed with ADM and CAM.

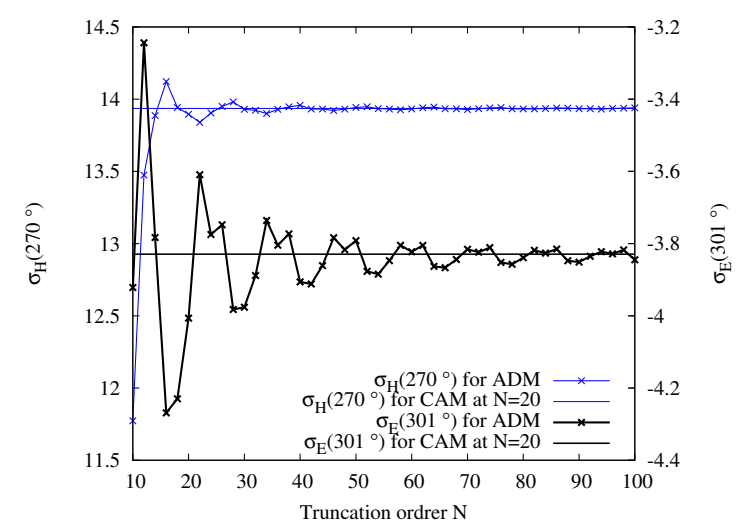

(b) Convergence tests of $\sigma_{H}\left(270^{\circ}\right)$ and $\sigma_{E}\left(301^{\circ}\right)$ versus truncation order $N$.

Fig. 5. (Color online) Comparison between DCS calculated with CAM and ADM for a biaxial medium. 


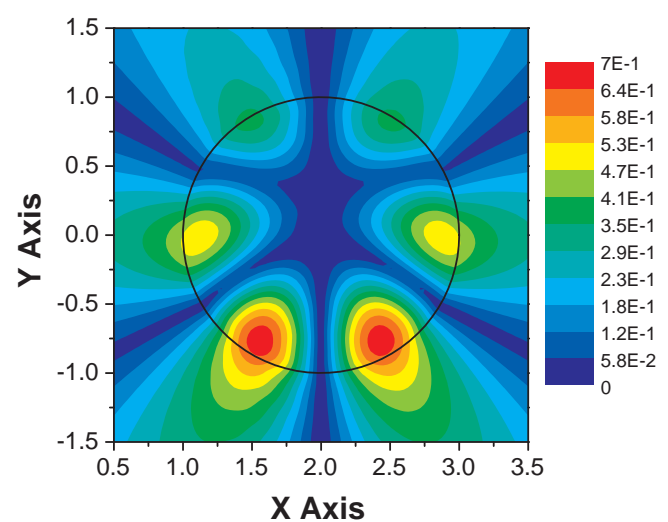

(a) $\left|E_{z}\right| \cdot \tau=0.17 \%$

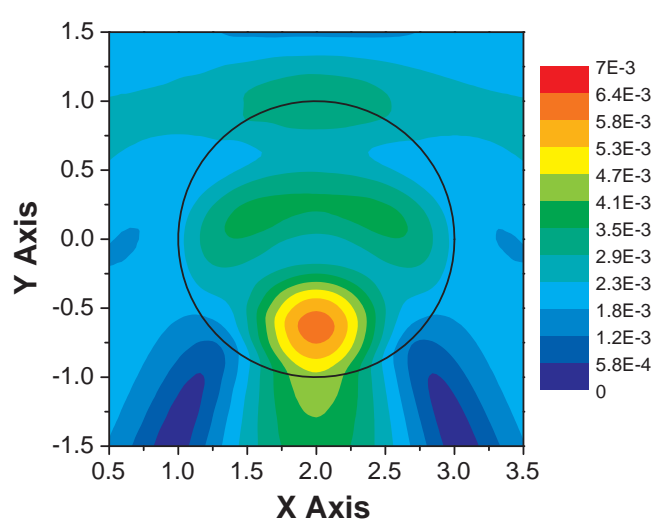

(b) $\left|H_{z}\right| \cdot \tau=0.022 \%$

Fig. 6. (Color online) Diffracted field maps for anisotropic circular cylinder computed with ADM for $N=60 . \tau$ is the relative error between CAM and MDA field maps (average relative error computed at each point of field maps). 


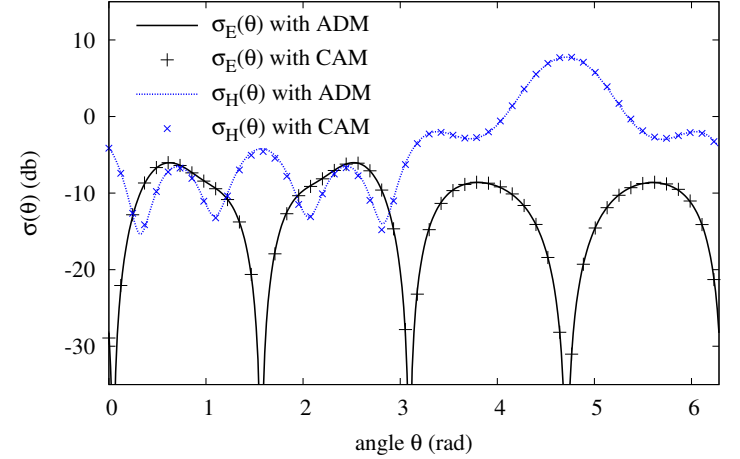

(a) DCS versus angle for electric and magnetic fields computed with ADM and CAM.

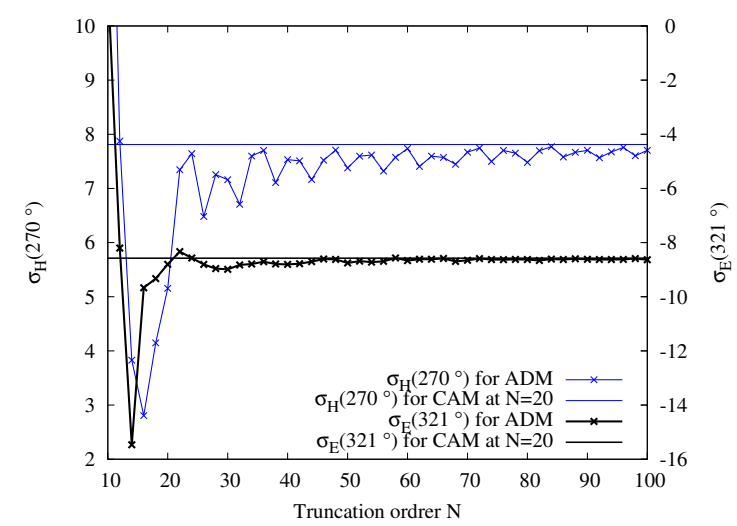

(b) Convergence tests of $\sigma_{H}\left(270^{\circ}\right)$ and $\sigma_{E}\left(321^{\circ}\right)$ versus truncation order $N$.

Fig. 7. (Color online) Comparison between DCS calculated with CAM and ADM for an unaxial medium. 


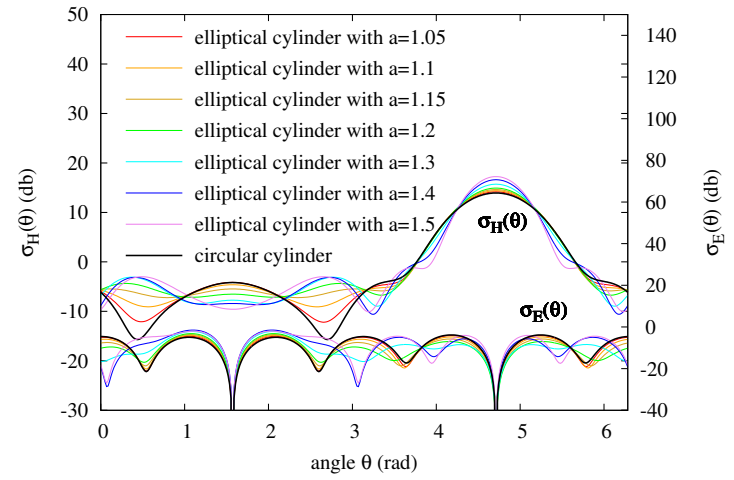

(a) DCS for anisotropic circular and elliptical cylinders

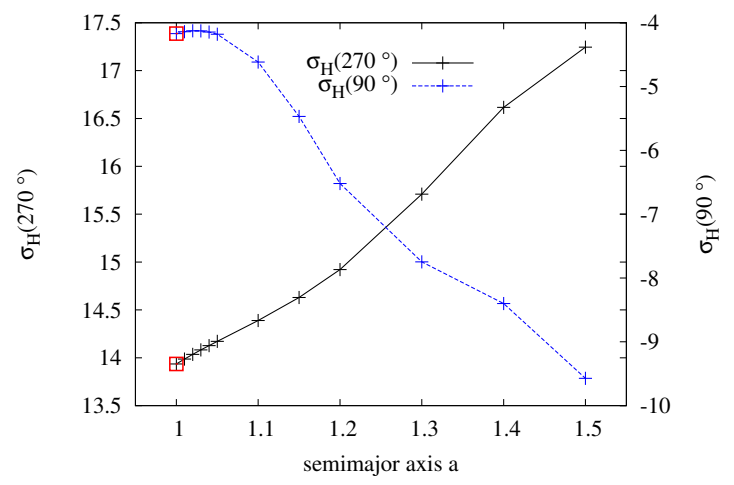

(b) Evolution of $\sigma_{H}\left(270^{\circ}\right)$ and $\sigma_{H}\left(90^{\circ}\right)$ versus $a$. The small boxes locate the value for the circular cylinder.

Fig. 8. (Color online) Study of DCS for anisotropic elliptical cylinders with different values of the semi-major axis $a$. The semi-minor axis $b$ is fixed at 1 . 


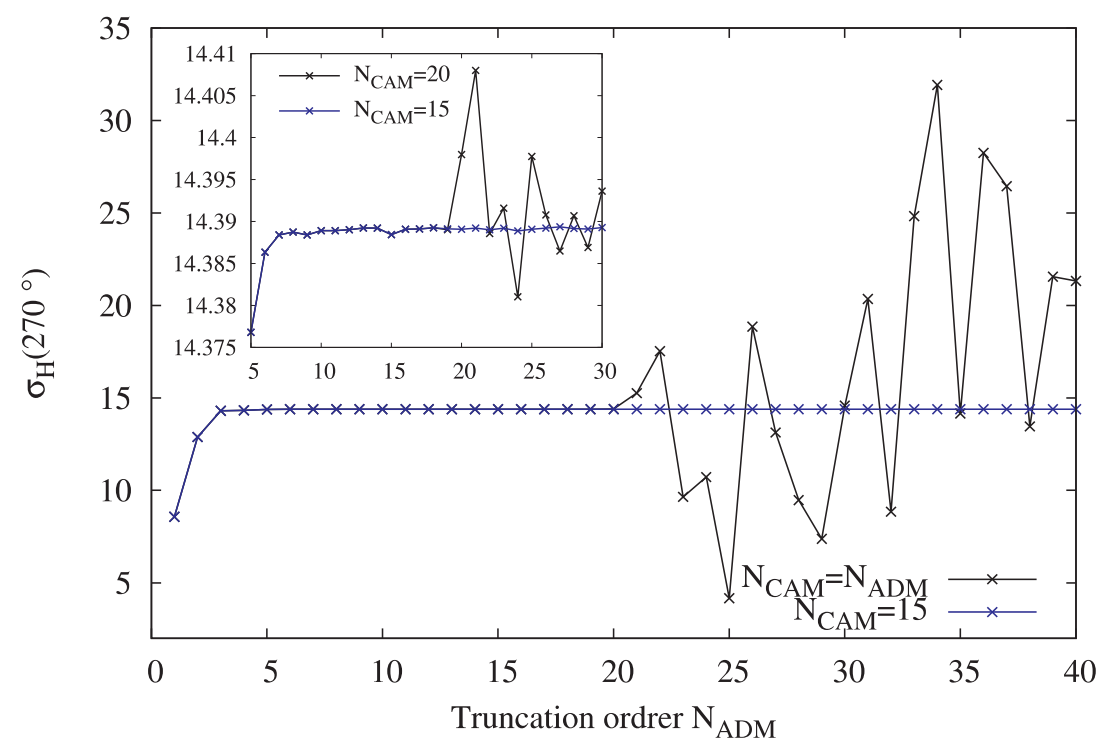

Fig. 9. (Color online) $\sigma_{H}\left(270^{\circ}\right)$ versus $N_{A D M}$ for different values of $N_{C A M}$ and for $a=1.1$. 